\title{
Fluctuation conductivity of inhomogeneous superconductors: A fractal aggregation model
}

\author{
M. A. Gusmão and P. M. Mors \\ Instituto de Física, Universidade Federal do Rio Grande do Sul, Caixa Postal 15051, 91500 Porto Alegre, \\ Rio Grande do Sul, Brazil
}

(Received 14 December 1989; revised manuscript received 26 July 1990)

\begin{abstract}
Numerical simulations of diffusion processes are performed on fractal aggregates designed to mimic the structure of inhomogeneous granular superconductors. The spectral dimensions obtained yield fluctuation-conductivity exponents that are compared with experimental results on ceramic samples of high- $T_{c}$ oxides of the $\mathrm{YBaCuO}$ system.
\end{abstract}

\section{INTRODUCTION}

The concept of fractal has lately received a great deal of attention, and applications of it have been devised in nearly all fields of physics and related sciences. ${ }^{1}$ Whenever inhomogeneities play a major role in determining the properties of a system it is almost certainly possible to advance a description in terms of fractals.

In the field of superconductivity, Char and Kapitulnik ${ }^{2}$ (CK) have pointed out the relevance of considering a fractal topology in order to explain the anomalous fluctuation conductivity observed in inhomogeneous superconducting materials. The excess conductivity $(\Delta \sigma)$ due to fluctuations into the superconducting state diverges as the transition temperature $T_{C}$ is approached from above, with a power law $^{3}$ of the form

$$
\Delta \sigma \sim \epsilon^{-\lambda},
$$

where $\epsilon \equiv\left(T-T_{C}\right) / T_{C}$ is the reduced temperature. For homogeneous superconductors the observed values of $\lambda$ generally agree with the Aslamazov-Larkin (AL) theory, ${ }^{4}$ which takes into account Gaussian fluctuations of the order parameter around the Ginzburg-Landau minimum. According to that theory, $\lambda$ is given in terms of the space dimension $d$ as $\lambda_{\mathrm{AL}}=\frac{1}{2}(4-d)$. The analysis of CK shows that for inhomogeneous materials the fracton dimension ${ }^{1}$ $\widetilde{d}$ (also called spectral dimension) of the underlying fractal structure should be substituted for the space dimension in $\lambda_{\mathrm{AL}}$ yielding

$$
\lambda=\frac{1}{2}(4-\widetilde{d}) \text {. }
$$

This has been successfully applied ${ }^{2}$ to the case of percolating clusters, which is experimentally realized in metal-insulator mixtures like Al-Ge.

It is well known that inhomogeneities play a major role in polycrystalline high- $T_{C}$ superconductors. In particular, a picture of superconducting grains connected by "weak links" is the usual explanation for the broad, twostep transition ${ }^{5}$ as well as the small critical currents ${ }^{6}$ that have been observed. Recent measurements ${ }^{7,8}$ of the fluctuation conductivity in ceramic samples of various high$T_{C}$ compounds have shown unequivocally the presence of inhomogeneity effects and the appearance of exponents not consistent with an integral dimensionality. The main experimental results of Ref. 8 may be summarized as follows.

(i) The temperature derivative of the resistivity presents two peaks, a feature that is consistent with the occurrence of a two-step transition in a granular system. The position of the higher-temperature peak (in general the sharpest one) is interpreted as the critical temperature $T_{C 1}$ at which a superconducting state is established inside the grains, while long-range order does not set in until the lower zero-resistance temperature $T_{C 0}$ is reached.

(ii) A power-law behavior as in Eq. (1) is observed in two different regimes. In the vicinity of the intragrain transition, where the reduced temperature is defined with respect to $T_{C 1}$, there appears a sample-dependent exponent $\lambda_{1}$, whose value increases from 1.0 to about 1.5 with increasing normal state resistivity (see Fig. 1). Near zero resistance, defining the reduced temperature with respect to $T_{C 0}$, the observed exponent, that we will call $\lambda_{0}$, has its values distributed with a small dispersion around 1.47 , not showing a significant sample dependence (Fig. 2). Another regime is observed further above $T_{C 1}$, and interpreted as a Maki-Thompson ${ }^{9}$ contribution (higher temperature part in Fig. 1). This will not be addressed here.

Although a thorough discussion of these results has been presented in Ref. 8 we include some comments here, for the sake of completeness. The observation of $\lambda_{1}$ close to 1.0 in low-resistance samples indicates that the dominant superconducting fluctuations are of a twodimensional nature, probably associated with the $\mathrm{Cu}-\mathrm{O}$ planes. The expected crossover to three dimensions ${ }^{10}$ apparently occurs very close to $T_{C 1}$, but it is masked by finite grain-size effects as well as the onset of intergrain correlations (smoothly curved behavior in the lower temperature part of Fig. 1). The increase in $\lambda_{1}$ with sample resistance may be interpreted as evidence that some kind of disorder, probably related to local variations of oxygen concentration, breaks the homogeneity of the superconducting fluctuations on the $\mathrm{Cu}-\mathrm{O}$ planes. Those fluctuations develop then in an effective dimension intermediate between one and two. A fractal topology for the global arrangement of grains and weak links can also be inferred from the value of $\lambda_{0}$. In this case, dominance of fluctuations in a larger length scale may explain the observed 


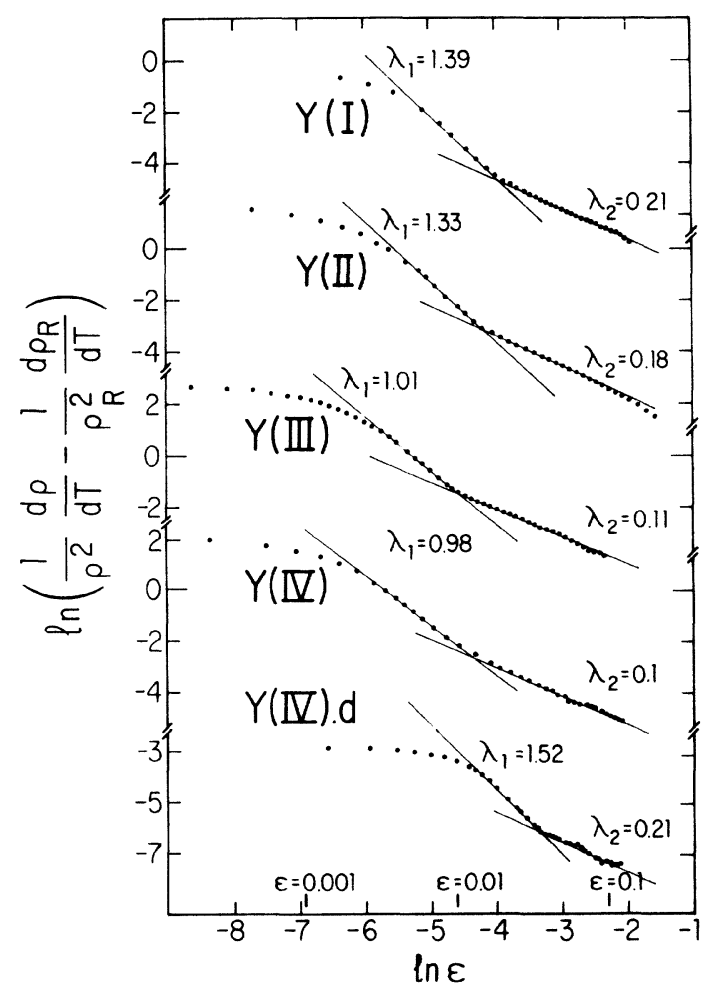

FIG. 1. Fluctuation contribution to the temperature derivative of the resistivity as a function of the reduced temperature near the intragrain transition, as reported in Ref. 8. Straight lines indicate power-law regimes for the fluctuation conductivity, in accordance with Eq. (1), with the quoted exponents. The $\lambda_{2}$ regime is not addressed in this work.

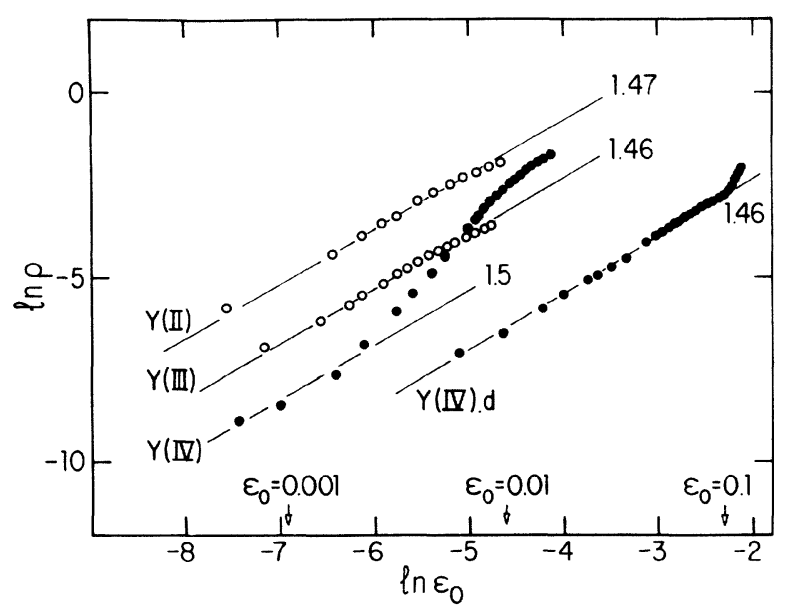

FIG. 2. Resistivity as a function of reduced temperature in the vicinity of the transition to zero resistance, as reported in Ref. 8. Continuous straight lines indicate power-law behavior with the quoted exponents. There is an early departure from the scaling behavior for one of the samples at higher temperatures, probably due to the small difference between $T_{C 1}$ and $T_{C 0}$ for that sample. sample independence, since one could expect all samples to be macroscopically identical.

Inspired on the above picture, we developed a numerical simulation of a simple model system showing a granular structure with fractal topology both globally and inside the grains. Its spectral dimension was determined (see Sec. III) by simulating a diffusion process (or random walk) in two regimes: (1) diffusion in a normal conductor and (2) diffusion in a random (fractal) network of superconducting grains and normal "bridges." The spectral dimensions so determined were related to the temperature ranges $T>T_{C 1}$ and $T_{C 0}<T<T_{C 1}$, respectively. We wish to emphasize that, in contrast to the fractal (or Hausdorff) dimension, ${ }^{1}$ which is a purely geometrical property, the spectral dimension depends on the effective topology for each process. For this reason, it has different values in each conduction regime.

We have built our system according to the clustercluster aggregation model for diffusion-limited aggregation (DLA), ${ }^{11,12}$ because it generates highly extended structures, whose dimensionality could be expected to fall in the range inferred from experiment. Due to the large amount of computational work involved, we have limited our simulations to two dimensions. Although this may be justified for the intragrain regime, a more realistic model of the approach to zero resistance should be based on a three-dimensional arrangement of grains and weak links.

In Sec. II we describe the construction of the aggregates and obtain their fractal dimension. The diffusion processes determining the relevant spectral dimensions and the corresponding fluctuation conductivity exponents are analyzed in Sec. III, and our main results are discussed in Sec. IV.

\section{CONSTRUCTION OF THE AGGREGATES}

Cluster-cluster (cl-cl) aggregation is a model for DLA that well succeeded in describing formation of fractal colloids and aerosols. ${ }^{12}$ Recently, it has been pointed out as a possible model for other observed fractal structures like, for instance, quantum-restricted semiconductor clusters. ${ }^{13}$ In its original version, cl-cl aggregation starts with a low concentration of identical (spherical) particles randomly distributed in a box, with periodic boundary conditions on the walls. At each step of the computation, one particle (or cluster) is chosen at random and made to perform a Brownian step of length equal to the particle diameter. Whenever two clusters touch, they stick together irreversibly, building up a new, larger cluster. Eventually a single final aggregate is formed. Such an object is a fractal ${ }^{1}$ characterized by a fractal dimension $D$, defined, as usual, through the relation between the object's mass, or number of particles in the aggregate $(N)$, and a typical linear size $R$ (the radius of gyration, for example):

$$
N \sim R^{D}
$$

For random fractals, as is the case of aggregates, $D$ must be determined after averaging over a large number of samples. 
The simulations are easier and faster if one uses a lattice version of the model, in which the only allowed positions for the particles are the sites of a (hipper) cubic lattice. The length of a Brownian step is now equal to the lattice parameter, and two clusters touch when at least one particle belonging to each of them occupy nearestneighbor positions. The fractal dimension of aggregates constructed according to the cl-cl model in twodimensional Euclidean space has been determined to be $1.44 \pm 0.05$, either on- or off-lattice. ${ }^{12}$

We worked out a generalization of the on-lattice cl-cl model in which two kinds of equal-sized particles are present in different proportions. Particles of one kind, in larger number, will eventually form the grains that are to become superconducting; the others will provide the "bridges" between those grains. During the growth process, whenever two clusters touch via particles of the same kind they stick together with probability one; when contact occurs through different types of particles the sticking probability is $p<1$. By choosing a low value for $p$, we ensure segregation of the two components and obtain the desired structure of grains and bridges in the final aggregate.

If we wanted to make a closer analogy between this model and the real materials, we could interpret the superconducting particles as representing small regions in the system in which inhomogeneity effects are not strong enough to influence superconducting correlations. Of course, the size of these regions depends strongly on the coherence length, which is actually very short in high- $T_{c}$ superconductors. On the other hand, the bridge particles in this model should represent the regions where the superconducting order parameter is strongly depleted, due to, for instance, variations in oxygen concentration. Here we are assuming that the size of the homogeneously coherent regions (superconducting particles) is approximately the same as that of depleted regions (bridge particles). Due to the higher concentration of superconducting particles, these will form large clusters (grains) that will eventually be connected to each other by small clusters of bridge particles (weak links). As discussed in Ref. 8 the superconducting grains are not necessarily related to the crystal structure and polycrystallinity, but only to conduction properties. We are also assuming that the volume fraction of material in which superconducting fluctuations actually develop in the vicinity of the transition is small. Otherwise, more compact structures, with higher fractal dimensions would be formed. Of course, this is a very simplified model, and its relation to real systems must be considered as pictorial.

Our simulations were performed in two dimensions, with 1000 particles on a square lattice of $158 \times 158$ sites, which corresponds to a global concentration of 0.04 . The relative concentrations of grain and bridge particles were chosen in the ratio 7:3, and we used a sticking probability $p=0.1$. The fractal dimension was determined in the following way. At each step of the growth process, the radius of gyration $(R)$ of the cluster with the largest number of particles $(N)$ was determined. These values were averaged over ten sample aggregates. The fractal dimension of this ten-sample "batch" was then determined by a

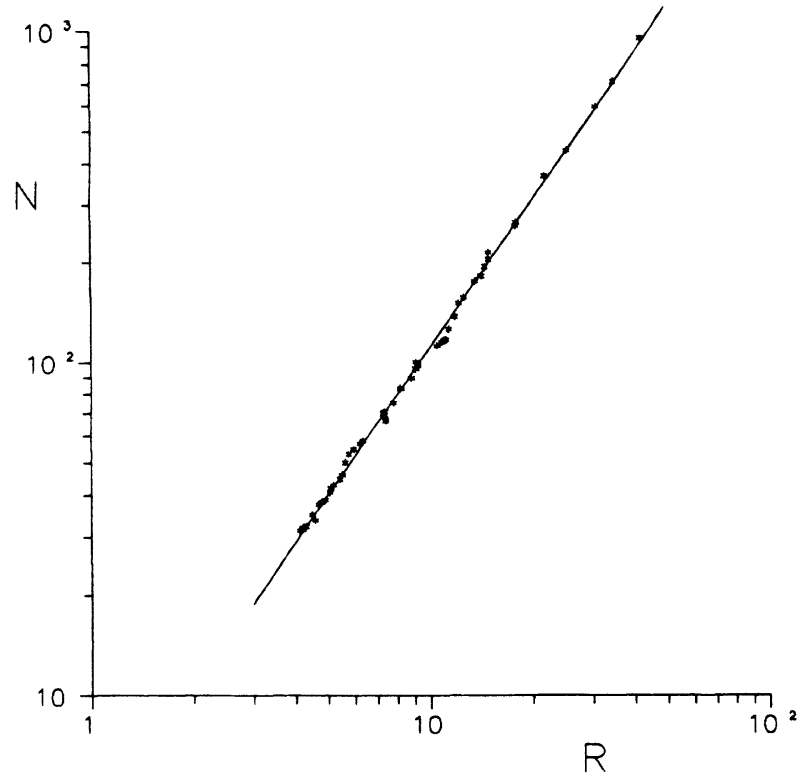

FIG. 3. Typical logarithmic plot of the number of particles in the largest cluster against the corresponding radius of gyration during the growth process, averaged over ten sample aggregates. The straight line is a linear fit to the data.

linear fit to a plot of $\log _{10} N \times \log _{10} R$, according to Eq. (3). A typical plot is shown in Fig. 3, where it can be seen that the agreement with Eq. (3) is very good. This program was run ten times and the final fractal dimension determined as the arithmetic mean of the fractal dimensions of the ten batches. The result was

$$
D=1.48 \pm 0.04 \text {. }
$$

Although the estimated uncertainty does not allow us to say that we have a significant difference with respect to the single component case, a slightly larger $D$ was actually expected, on the basis that lower sticking probabilities tend to generate more compact aggregates. This has been verified in some extensions of the cl-cl model. ${ }^{12}$

\section{DETERMINATION OF THE SPECTRAL DIMENSIONS}

The spectral dimension of a fractal structure may be obtained by studying diffusion processes in that structure. ${ }^{12}$ It is well known that the mean-square-distance of a random walker to its starting position grows linearly with time (for long times) when the random walk is performed on compact lattices, independently of their dimensionality. On fractal lattices, however, this linearity breaks down, and anomalous diffusion occurs. The mean-square-distance of the random walker now depends on the spectral and fractal dimensions of the lattice according to the relation ${ }^{12}$

$$
\left\langle r^{2}(t)\right\rangle \sim t^{d / D} .
$$

In our model we have two spectral dimensions, depending on whether we are studying diffusion in the normal state $\left(\lambda_{1}\right.$ regime $)$ or in the fractal superconducting 
network state $\left(\lambda_{0}\right.$ regime). The simulation for the $\lambda_{1}$ regime is just a random walk on a fractal lattice, or the "ant in the labyrinth" model, ${ }^{14}$ since there is no significant difference between the particles that constitute the aggregate in this situation. For the $\lambda_{0}$ regime, though, a special kind of random walk is needed in which the speed of the walker becomes infinite inside the superconducting grains while remaining finite on the bridges. This is indeed the "termite diffusion" model, ${ }^{15}$ that has been used to analyze random superconducting networks. In practice, the simulation is performed with the time counter being incremented only when the walker steps on a bridge particle.

We averaged $r^{2}(t)$ over 15000 walks on each 10 sample aggregates. To avoid surface effects, each walker started on the particle closest to the center of mass of the aggregate. In the end, the values of $\left\langle r^{2}(t)\right\rangle$ for each time were again averaged over the ten samples. The results are plotted in Fig. 4. It can be seen that the asymptotic regime is rapidly reached, and the agreement with Eq. (4) is very good. The fracton dimensions obtained from those plots for the two regimes considered above are

$$
\widetilde{d}_{1}=1.11 \pm 0.03
$$

and

$$
\widetilde{d}_{0}=0.92 \pm 0.03 \text {. }
$$

With these, and using CK's prescription, Eq. (2), we determined the fluctuation conductivity exponents relevant to each regime to be

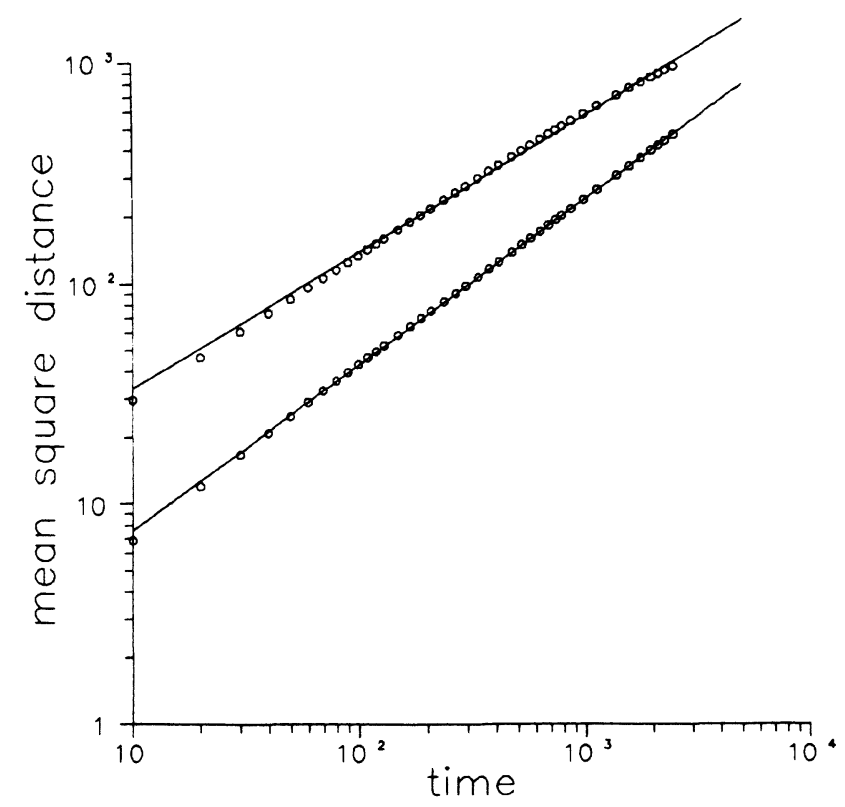

FIG. 4. Logarithmic plot of the mean-square distance of a random walker to its starting position as a function of "time." The upper (lower) straight line is a linear fit to the data obtained for the $\lambda_{0}\left(\lambda_{1}\right)$ regime. The density of data points for large times is much higher than shown in the figure, where it has been reduced for the sake of clarity. The fittings have been performed with the complete data set.

$$
\lambda_{1}=1.45 \pm 0.02
$$

and

$$
\lambda_{0}=1.54 \pm 0.02 .
$$

Comparing these values with the experimental results referred to in Sec. I, one can see that the agreement between our simulation for the $\lambda_{1}$ regime and the experimental values for high resistance samples is quite good. In addition, the observed relationship $\lambda_{1}<\lambda_{0}$ is qualitatively preserved.

\section{CONCLUSIONS}

We have presented a model simulation of an inhomogeneous granular superconducting network in two dimensions, constructed within the cluster-cluster aggregation scheme. Two kinds of particles, with a low sticking probability between different particles, yielded the formation of inhomogeneous superconducting grains interconnected by normal weak links. Numerical simulation of diffusion processes in these aggregates, both in the normal state and in the weak-linked superconducting grains regime, were used to determine fracton dimensions for both cases. These, in turn, were employed to determine fluctuation conductivity exponents, through the relation obtained by Char and Kapitulnik's modification of the Aslamazov-Larkin theory for the inhomogeneous case.

Our results are of interest in two distinct areas. As far as fractal growth is concerned we have constructed a new type of aggregate, with two kinds of equal-sized particles that segregate during the growth process, forming a granular fractal structure. We have then determined its fractal and spectral dimensions. There might be real systems other than ceramic superconductors that can be modeled according to this scheme. On the superconductivity side, our results reproduce qualitatively - and, to a limited extent, quantitatively - the experimental observations of fluctuation conductivity exponents, providing insight on the topology of the superconducting state in high- $T_{C}$ oxides.

We obviously do not claim to have a realistic model of the polycrystalline oxide superconductors. Nevertheless, we can say that some features of our "toy" model have a counterpart in the real system. We could not expect good agreement with experiment as far as the $\lambda_{0}$ regime is concerned, since our simulations are restricted to two dimensions, while intergrain correlations in the real system certainly take place in three-dimensional space. We can, however, have higher expectations concerning the $\lambda_{1}$ regime, on the basis that two-dimensional processes occurring on the $\mathrm{Cu}-\mathrm{O}$ planes could be expected to dominate the system's behavior. In this case, the quantitative agreement obtained may be indicative that short-scale inhomogeneities in high-resistance samples of $\mathrm{YBa}_{2} \mathrm{Cu}_{3} \mathrm{O}_{7}$ and related compounds prevent superconducting fluctuations to develop uniformly in the $\mathrm{Cu}-\mathrm{O}$ planes. Furthermore, it may indicate that the topology of the fraction of those planes on which the superconducting state is actually established may be similar to the one shown by fractal aggregates constructed by the cl-cl model in two di- 
mensions. Experimentally it was observed ${ }^{8}$ that the exponent $\lambda_{1}$ can vary significantly from sample to sample, with a range of values consistent with topology variations from homogeneous planes to percolation clusters $\left(\widetilde{d}=\frac{4}{3}\right)$ to fractal aggregates. Our choice of the last one was a matter of convenience, in order to study the topological crossover between the two regimes.

Other questions may be raised concerning the nearzero resistance regime, besides the restriction to two dimensions in this simulation. For instance, it is not clear that our "bridges" can be a meaningful representation of the actual weak links. Here we just kept them in the normal state below the critical temperature for the intragrain transition. The weak links in the real materials are most probably Josephson junctions. ${ }^{16}$ However, we believe that this would not invalidate the simulations, since a tunneling probability across the junction would manifest itself in a random "time delay" to cross the bridge, which is essentially what happens in our model.

Of course, the present analysis would not apply, as discussed in Ref. 8, if critical (non-Gaussian) fluctuations were important, in which case a simple relation like Eq. (2) would break down. Nevertheless, we insist on the relevance of fractal topology effects, based on the experimental evidence of nonuniversal conductivity exponents, a fact that could not be explained in the framework of the theories of critical phenomena. The situation is not so clear with respect to the approach to zero resistance, since there the experiments reveal a nearly universal behavior. One would thus be tempted to discard an ex- planation in terms of a fractal geometry. However, such a picture may still hold if one considers the universal behavior as reflecting a macroscopic similarity between samples, in a regime where long-range correlations are dominant. Obviously, in a still larger length scale the system should look homogeneous. We do not believe, though, that a crossover to this situation could be experimentally discernible in the high- $T_{C}$ materials if we restrict ourselves to resistivity measurements. The reason for this is that the relevant length scale for conductivity fluctuations is the coherence length $\xi_{c}$, which is very short in these compounds. Homogeneous behavior would be expected only if $\xi_{c}$ were larger than the characteristic length of the inhomogeneities. This could be observed if a true critical region were experimentally accessible, since in this case the coherence length should increase near $T_{C}$. Of course, our model aggregates are fractal by construction, and any comparison with experiment must be based on the assumption that the coherence length is smaller than the characteristic length scale of the inhomogeneities in the range of temperatures investigated.

\section{ACKNOWLEDGMENTS}

We thank P. Pureur for enlightening discussions in the early stages of this work. We also acknowledge financial support from Conselho Nacional de Desenvolvimento Cientifico e Tecnológico (CNPq) and Financiadora de Estudos e Projectos (FINEP), Brazil.
${ }^{1}$ B. B. Mandelbrot, Fractal Geometry of Nature (Freeman, San Francisco, 1983).

${ }^{2}$ K. Char and A. Kapitulnik, Z. Phys. B Condensed Matter 72, 253 (1988).

${ }^{3}$ M. J. Skocpol and M. Tinkham, Rep. Prog. Phys. 38, 1049 (1975).

${ }^{4}$ L. G. Aslamazov and A. I. Larkin, Sov. Phys. Solid State 10, 875 (1968).

${ }^{5}$ P. Pureur and J. Schaf, J. Magn. Magn. Mater. 69, L215 (1987).

${ }^{6}$ J. W. Ekin, A. I. Braginski, A. J. Panson, M. A. Janocko, D. W. Capone II, W. J. Zaluzec, B. Flandermayer, O. F. de Lima, M. Hong, J. Kuo, and S. H. Liou, J. Appl. Phys. 62, 4821 (1987).

${ }^{7}$ M. Ausloos, P. Clippe, and Ch. Laurent, Phys. Rev. B 41, 9506 (1990).

${ }^{8}$ P. Pureur, J. Schaf, M. A. Gusmão, and J. V. Kunzler, Progress in High Temperature Superconductivity, edited by R. Nicolsky (World Scientific, Singapore, 1990), Vol. 25, pp. 236-244.
${ }^{9}$ K. Maki, Prog. Theor. Phys. (Japan) 39, 897 (1968); R. S. Thompson, Phys. Rev. B 1, 327 (1970).

${ }^{10} \mathrm{~W}$. E. Lawrence and S. Doniach, in Proceedings of the 12th International Conference on Low Temperature Physics-Kyoto, 1970, edited by E. Kanda (Keigaku, Tokio, 1971), p. 361.

${ }^{11}$ P. Meakin, Phys. Rev. Lett. 51, 1119 (1983); M. Kolb, R. Botet, and R. Jullilen, ibid. 51, 1123 (1983).

${ }^{12}$ R. Jullien and R. Botet, Aggregation and Fractal Aggregates (World Scientific, Singapore, 1987); R. Jullien, R. Botet, and P. M. Mors, Discuss. Faraday Chem. Soc. 83, 125 (1987); R. Botet and R. Jullien, Ann. Phys. (Paris) 13, 153 (1988).

${ }^{13}$ M. O'Neil and G. McLendon, Chem. Phys. Lett. 147, 565 (1988).

${ }^{14}$ P. G. de Gennes, Recherche 7, 919 (1976).

${ }^{15}$ P. G. de Gennes, J. Phys. Colloq. 41, C3 (1980).

${ }^{16}$ G. Deutscher, Physica C 153 - 155, 15 (1988); R. L. Peterson and J. W. Ekin, Phys. Rev. B 37, 9848 (1988). 